7. Y. C. Wong, Existence of linear connections with respect to which given tensor fields are parallel or recurrent, Nagoya Math. J. 24 (1964), 67-108.

NORTHWESTERN UNIVERSITY

\title{
A NOTE ON A REDUCIBLE CONTINUUM
}

\section{E. L. BETHEL}

In [4], Knaster shows that there exists an irreducible compact metric continuum $M$ which has a monotone continuous decomposition $G$ such that each element of $G$ is nondegenerate and $M / G$ is an arc. Also, he raised the question as to whether there existed an irreducible continuum $M$ which has a monotone continuous decomposition $G$ such that each element of $G$ is an arc and $M / G$ is an arc. E. E. Moise settled this question in the negative in [5]. In [3], M. E. Hamstrom showed that if $G$ is a monotone continuous decomposition of a compact metric continuum such that each element of $G$ is a nondegenerate continuous curve and $M / G$ is an arc, then it is not the case that $M$ is irreducible. E. Dyer generalized this result by showing in [2] that if $M$ is a compact metric continuum and $G$ is a monotone continuous decomposition of $M$ such that each element of $G$ is nondegenerate and decomposable, then it is not the case that $M$ is irreducible. A purpose of this note is to extend Dyer's result somewhat.

The author is indebted to the referee for some suggestions which have been incorporated in this note. In particular, a weakened hypothesis in Theorem 2.

THEOREM 1. Let $M$ denote a compact metric continuum and $G$ a nondegenerate monotone continuous decomposition of $M$ each of whose elements is nondegenerate. If $H$ is a subcollection of $G$ each of whose elements is snakelike and indecomposable, and if $H^{*}$ is dense in $M$, then uncountably many elements of $G$ are indecomposable.

Proof. Let $I_{1}$ denote an element of $H$, and let $C_{1}$ denote the first chain in a sequence of defining chains for $I_{1}$, and let $L_{1}$ and $L_{2}$ denote the end links of $C_{1}$. Since $H^{*}$ is dense in $M$, and $G$ is a continuous collection, $C_{1}$ contains two elements $I(10)$ and $I(11)$ of $H$ such that $I(10)$ and $I(11)$ intersects every link of $C_{1}$. Let $\left\{C_{n}(10)\right\}$ and $\left\{C_{n}(11)\right\}$ denote chain sequences which define $I(10)$ and $I(11)$ respectively.

Presented to the Society, November 13, 1965 under the title On reducibility of continua; received by the editors January 11, 1965. 
It follows that there is some $C_{i}(10)$ of $\left\{C_{n}(10)\right\}$ and some $C_{j}(11)$ of $\left\{C_{n}(11)\right\}$ such that (1) each is a refinement of $C_{1},(2)$ each has links intersecting the first and last links of $C_{1}$, and (3) there exist points $A_{1}$ and $A_{2}$ of $L_{1}$ and $L_{2}$ respectively, such that if $U$ is a coherent two region collection of either $C_{i}(10)$ or $C_{j}(11)$ which intersects $A_{i}$, $(i=1,2)$, then $U^{*} C L_{i},(i=1,2)$. Furthermore, the closure of the union of both chains does not intersect $I_{1}$, and the diameter of each link is less than $1 / 2$. By Theorem 2 of [1], there is some $C(10)$ of $\left\{C_{n}(10)\right\}$ and a $C(11)$ of $\left\{C_{n}(11)\right\}$ such that $C(10)$ and $C(11)$ each is a refinement of and loop in $C_{i}(10)$ and $C_{j}(11)$ respectively. It is an easy exercise to show that both $C(10)$ and $C(11)$ loop in $C_{1}$ also.

Now repeat the process used for the construction of $C(10)$ and $C(11)$ in both $C(10)$ and $C(11)$, where $C(10)$ and $C(11)$ assume the role of $C_{1}$ and each of $I(10)$ and $I(11)$ assume the role of $I_{1}$. By induction, we may define a sequence of chains $\left\{C\left(i_{1} \cdots i_{n}\right)\right\},\left(i_{k}=0,1\right)$, such that (1) $I\left(i_{1} \cdots i_{n}\right)$ is an element of $H$ which is a subset of the union of the links of $C\left(i_{1} \cdots i_{n}\right)$ and intersects each link of $C\left(i_{1} \cdots i_{n}\right)$, (2) $C\left(i_{1} \cdots i_{n} k\right),(k=0,1)$, loops and is a refinement of $C\left(i_{1} \cdots i_{n}\right)$, and (3) each link of $C\left(i_{1} \cdots i_{n}\right)$ has diameter less than $1 / n$. Thus, each sequence $\left\{i_{n}\right\},\left(i_{n}=0,1\right)$, defines a sequence of chains such that the common part of the sequence of chains is an indecomposable continuum $I$ by Theorem 2 of [1]. Since $I\left(i_{1} \cdots i_{n}\right)$ intersects each link of $C\left(i_{1} \cdots i_{n}\right)$, we have a sequence of elements of $G$ converging to $I$, and $I \cap I\left(i_{1} \cdots i_{n}\right)=\varnothing$, it follows that $I \in G$. Since there are uncountably many sequences $\left\{i_{n}\right\}, G$ contains uncountably many snakelike indecomposable continua.

Theorem 2. Let $M$ denote a compact irreducible continuum, and let $G$ be a nondegenerate monotone continuous decomposition of $M$ each of whose elements is nondegenerate and either snakelike or decomposable. If $M / G$ has a dense set of separating points, then uncountably many elements of $G$ are indecomposable.

Proof. Suppose the contrary. Let $G^{\prime}$ denote the elements of $G$ which are indecomposable and suppose $G^{\prime}$ is countable. Now $\left(G^{\prime}\right)^{*}$ is not dense in $M$ since this would imply that $G^{\prime}$ is uncountable by Theorem 1. Let $A$ and $B$ denote two points between which $M$ is irreducible, and let $g$ denote a separating element of $G$ which does not belong to $G^{\prime}$. There exists an open set $D$ with respect to $M / G$ containing $g$ such that $\bar{D} \cap\left(H \cup g_{A} \cup g_{B}\right)=\varnothing$, where $g_{A}$ and $g_{B}$ are the elements of $G$ containing $A$ and $B$ respectively. There is some subcontinuum $K$ of $M / G$ such that $K$ is irreducible from $M / G-D$ to $g$. It follows that each element of $K$ is decomposable. Since the set of 
separating points are dense in $M / G$, there is a separating point $g^{\prime}$ of $K$ distinct from $g$. Furthermore, there is a subcontinuum $K^{\prime}$ of $K$ irreducible from $g^{\prime}$ to $g$. But since each element of $K^{\prime}$ is decomposable, by Dyer's theorem, there is a proper subcontinuum $L$ of $\left(K^{\prime}\right)^{*}$ intersecting $g$ and $g^{\prime}$. Since $g$ and $g^{\prime}$ are separating points of $M / G$, it now easily follows that $M$ is not irreducible from $A$ to $B$, a contradiction. Hence, uncountably many elements of $G$ are indecomposable.

REMARK. May the stipulation that $M / G$ has a dense set of separating points be removed or replaced by a weaker stipulation? Indeed, may the stipulation that the indecomposable elements be snakelike be removed?

\section{REFERENCES}

1. L. K. Barrett, The structure of decomposable snakelike continua, Duke Math. J. 28 (1961), 515-521.

2. E. Dyer, Irreducibility of the sum of the elements of a continuous collection of continua, Duke Math. J. 20 (1953), 589.

3. M. E. Hamstrom, Continuous collections of continuous curves, Bull. Amer. Math. Soc. 58 (1952), 204.

4. B. Knaster, Un continua irreductible d decomposition continue en tranches, Fund. Math. 25 (1953), 568-577.

5. E. E. Moise, Monotone interior transformations; Bull. Amer. Math. Soc. 55 (1949), 810-811.

Clemson University 